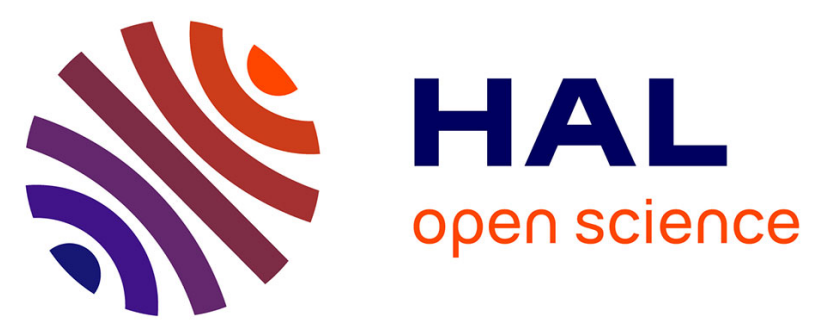

\title{
AN ILLUMINANT-INDEPENDENT ANALYSIS OF REFLECTANCE AS SENSED BY HUMANS, AND ITS APPLICABILITY TO COMPUTER VISION
}

Alban Flachot, Edoardo Provenzi, J Kevin O'Regan

\section{- To cite this version:}

Alban Flachot, Edoardo Provenzi, J Kevin O'Regan. AN ILLUMINANT-INDEPENDENT ANALYSIS OF REFLECTANCE AS SENSED BY HUMANS, AND ITS APPLICABILITY TO COMPUTER VISION. EUVIP, Oct 2016, Marseille, France. hal-01324912

\section{HAL Id: hal-01324912 \\ https://hal.science/hal-01324912}

Submitted on 1 Jun 2016

HAL is a multi-disciplinary open access archive for the deposit and dissemination of scientific research documents, whether they are published or not. The documents may come from teaching and research institutions in France or abroad, or from public or private research centers.
L'archive ouverte pluridisciplinaire HAL, est destinée au dépôt et à la diffusion de documents scientifiques de niveau recherche, publiés ou non, émanant des établissements d'enseignement et de recherche français ou étrangers, des laboratoires publics ou privés. 


\section{AN ILLUMINANT-INDEPENDENT ANALYSIS OF REFLECTANCE AS SENSED BY HUMANS, AND ITS APPLICABILITY TO COMPUTER VISION}

\author{
Alban Flachot, \\ Phelma, INP Greoble \\ Master IC ${ }^{2}$ A Sciences Cognitives \\ 3 Parvis Louis Nel - CS 50257 - 38016 \\ Grenoble Cedex 01, France
}

* Edoardo Provenzi, ** J.Kevin O'Regan

\author{
Universite Paris Descartes, \\ * Laboratoire MAP5 \\ ** Laboratoire LPP \\ 45 rue des Saints Peres 75006 Paris, France
}

\begin{abstract}
Philipona \& O'Regan (2006) [1] recently proposed a linear model of surface reflectance as it is sensed by the human eyes. In their model, the tristimulus response to reflected light is accurately approximated by a linear transformation of the tristimulus response to illumination, allowing the prediction of several perceptual characteristics of human vision. Later, Vazquez-Corral et al (2012) [2] built a bridge between Philipona \& O'Regan's model and von Kries-like approaches to color constancy in computer vision by showing that the linear operators could be diagonalized in a common basis. However both of these studies required specifying a particular dataset of illuminants. We will show in this paper that it is possible to compute adequate linear operators and a common basis for diagonalization without specifying any particular set of illuminants.
\end{abstract}

Index Terms - Philipona-O'Regan's model, Illuminant independency, Joint diagonality, von Kries model.

\section{INTRODUCTION}

Philipona \& O'Regan (2006) [1] (PO from now on) recently proposed a linear model of surface properties as they are sensed by the human eyes. A crucial point of the model is the definition of a linear operator in the cone response space as being responsible for the transformation, by the surface, of the perceived incident light into the perceived reflected light. Their model is able to predict perceptual characteristics of human color vision, as unique hues and hue cancelation. Later, Vazquez-Corral et al (2012) [2] (V-C from now on) were able to build a bridge between PO's model and classic strategies to achieve color constancy in computer vision, as the von Kries diagonal method. They did this by computing a global transformation in which all of the linear operators defined by PO can be approximately considered diagonal. However, both of these studies rely on the specification of a data set of natural illuminants. This limits their robustness with respect to every other kind of illuminant. Furthermore,
V-C strategy relies on the designation of a particular standard illuminant. We will show in this paper that it is possible to compute linear operators and also to make a global change of basis without resorting to any database of illuminants.

This paper is divided in two parts: in a first part, we will introduce PO's model in detail and present a novel computational/statistical approach to the model that is completely illuminant-independent. A quantitative comparison will be discussed between the two. In a second part, we will start by explaining thoroughly how the link between PO's model and classical strategies to achieve color constancy can be made through the definition of a global diagonalizing matrix. Then, we will use theoretical and computational arguments to justify the existence of such a surface and illuminant-independent transformation and finish by proposing a new method to compute this transformation.

\section{PHILIPONA \& O'REGAN'S MODEL}

\subsection{Biological analogy of a surface reflectance}

Consider a surface $\mathbb{S}$ illuminated by a light with spectral power distribution $E(\lambda), \lambda \in \Lambda=[400,700] \mathrm{nm}, \Lambda$ being the visual spectrum. Let $R_{L}(\lambda), R_{M}(\lambda), R_{S}(\lambda)$ denote the absorption rate at each wavelength $\lambda$ by photopigments present in the $L, M, S$ human photoreceptors. The information accessible to the human nervous system can be expressed by $\mathbf{u}(E)=\left(u_{L}(E), u_{M}(E), u_{S}(E)\right)^{t}$, where:

$$
u_{i}(E)=\int_{\Lambda} R_{i}(\lambda) E(\lambda) d \lambda, \quad i=L, M, S .
$$

If we denote with $S(\lambda)$ the reflectance function of the surface $\mathbb{S}$, we can describe the accessible information about the light reflected by $\mathbb{S}$ as $\mathbf{v}^{\mathbb{S}}(E)=\left(v_{L}^{\mathbb{S}}(E), v_{M}^{\mathbb{S}}(E), v_{S}^{\mathbb{S}}(E)\right)^{t}$, with:

$$
v_{i}^{\mathbb{S}}(E)=\int_{\Lambda} R_{i}(\lambda) E(\lambda) S(\lambda) d \lambda, \quad i=L, M, S,
$$

we stress that this equation holds true only if there is no exchange of energy between different wavelengths (e.g. it does 
not hold for fluorescent surfaces). PO hypothesized that the transformation of $\mathbf{u}(E)$ into $\mathbf{v}^{\mathbb{S}}(E)$ is linear i.e.:

$$
\mathbf{v}^{\mathbb{S}}(E)=A^{\mathbb{S}} \mathbf{u}(E),
$$

where $A^{\mathbb{S}}: \mathbb{R}^{3} \mapsto \mathbb{R}^{3}$ is a linear operator that can be represented by a $3 \times 3$ matrix and which should only depend on the surface $\mathbb{S}$ and not on the illuminant $E$. We interpret $A^{\mathbb{S}}$ as the biological analogue of the physical reflectance of a surface, since it is the operator responsible for the transformation by a surface of the incident light into the reflected light, as sensed by the photoreceptors. We will thus call $A^{\mathbb{S}}$ the reflectance matrix (RM from now on).

The assumption of the existence of such a linear operator is not trivial. PO used statistical tools in order to verify it: they computed the empirical variance of the residuals $\eta=\mathbf{v}^{\mathbb{S}}(E)-$ $A^{\mathbb{S}} \mathbf{u}(E)$, where $A^{\mathbb{S}}$ is obtained by linear regression of $\mathbf{v}^{\mathbb{S}}$ onto $u$ over a set of natural and simulated daylight illuminants [1]. The coefficients of linear determination $R^{2}$ for their set of surfaces (over 1600 Munsell chips and 1800 natural surfaces) are surprisingly high, with mean values higher than 0.995, which shows the validity of the linear hypothesis.

We notice that, with this method, the matrix $A^{\mathbb{S}}$ depends on the dataset of illuminants used to compute it. In Section 3 we will discuss in greater detail this dependence and present a novel method for the computation of the RMs that allows complete independence with respect to the illuminant.

\subsection{Diagonalisation of the reflectance matrix and compu- tation of the virtual basis}

PO showed that for every surface of their database, the RM $A^{\mathbb{S}}$ can either be exactly diagonalized or almost diagonalized with three distinct real eigenvalues. In other words, there exists an invertible matrix $T^{\mathbb{S}}$, which depends on the surface $\mathbb{S}$, such that:

$$
\mathbf{v}^{\mathbb{S}}(E)=\left(T^{\mathbb{S}}\right)^{-1} D^{\mathbb{S}} T^{\mathbb{S}} \mathbf{u}(E),
$$

where $D^{\mathbb{S}}$ is a diagonal $3 \times 3$ matrix. $T^{\mathbb{S}}: \mathbb{R}^{3} \rightarrow \mathbb{R}^{3}$ is then the linear operator responsible for the change of basis where $A^{\mathbb{S}}$ is diagonal. This basis is given by the eigenvectors of $A^{\mathbb{S}}$.

Equation (4) can be rewritten as:

$$
\begin{aligned}
T^{\mathbb{S}} \mathbf{v}^{\mathbb{S}}(E) & =D^{\mathbb{S}} T^{\mathbb{S}} \mathbf{u}(E) \\
\tilde{\mathbf{v}}^{\mathbb{S}}(E) & =D^{\mathbb{S}} \tilde{\mathbf{u}}^{\mathbb{S}}(E),
\end{aligned}
$$

where $\tilde{\mathbf{u}}(E)$ and $\tilde{\mathbf{v}}^{\mathbb{S}}(E)$ are vectors in the new basis obtained by applying the operator $T^{\mathbb{S}}$. Note that, since $T^{\mathbb{S}}$ depends on the surface $\mathbb{S}$, so does $\tilde{\mathbf{u}}^{\mathbb{S}}(E)$.

By linearity and recalling Eq. (1), we can write $T^{\mathbb{S}} u_{i}(E)=$ $\int_{\Lambda} \sum_{j} T^{\mathbb{S}}{ }_{i j} R_{j}(\lambda) E(\lambda) d \lambda$, for every $i, j=L, M, S$. If we define $\tilde{R}_{i}=\sum_{j=L, M, S} T^{\mathbb{S}}{ }_{i j} R_{j}(\lambda)$, we find:

$$
\tilde{u}_{i}^{\mathbb{S}}(E)=\int_{\Lambda} \tilde{R}_{i}(\lambda) E(\lambda) d \lambda, \quad i=L, M, S,
$$

and likewise for $\tilde{v}_{i}^{\mathbb{S}}(E)$.

V-C [2] have interpreted $\tilde{R}_{i}$ as the absorption rate at each wavelength $\lambda$ of virtual photopigments present in virtual photoreceptors. For this reason, the basis in which the RM is diagonal is called the virtual basis, denoted by $\tilde{L} \tilde{M} \tilde{S}^{\mathbb{S}}$. The diagonality of the RM implies that the components of $\tilde{u}^{\mathbb{S}}(E)$ do not mutually interact with each other when they are reflected by the surface $\mathbb{S}$, and are simply individually scaled by the associated eigenvalues. In other words, the absorption rate curves of the virtual photopigments are less overlapping than the real ones.

Then, by definition of the virtual basis we have $\tilde{v}_{i}^{\mathbb{S}}(E)=$ $r_{i}^{\mathbb{S}} \tilde{u}_{i}^{\mathbb{S}}(E), i=\tilde{L}, \tilde{M}, \tilde{S}$, which is equivalent to:

$$
r_{i}^{\mathbb{S}}=\frac{\tilde{v}_{i}^{\mathbb{S}}(E)}{\tilde{u}_{i}^{\mathbb{S}}(E)} .
$$

$r_{\tilde{L}}^{\mathbb{S}}, r_{\tilde{M}}^{\mathbb{S}}, r_{\tilde{S}}^{\mathbb{S}}$ can thus be simply interpreted as three independent reflection coefficients in each of the three virtual channels.

\section{ANALYSIS OF THE INDEPENDENCE OF PHILIPONA \& O'REGAN'S LINEAR HYPOTHESIS WITH RESPECT TO THE ILLUMINANT}

As said previously in Section 2.1, the method used by PO to compute the RM is intrinsically dependent on the database of illuminants it relies on. As a consequence, the computed RMs will not accurately apply to an illuminant $E$ sharing poor similarities with the database used for the linear regression. In this section, we will discuss a procedure to compute the matrices $A^{\mathbb{S}}$ without resorting to any particular database of illuminants. We will see that the technique we propose is a good trade-off between robustness with respect to illuminant changes and the consistency of the matrices $A^{\mathbb{S}}$ with the linear model expressed by Eq. (3).

\subsection{A novel approach to compute the RMs independently with respect to the illuminant}

Let $A_{i j}^{\mathbb{S}}, j=L, M, S$, denote the components of $A^{\mathbb{S}}$. Then, if the linear hypothesis of $\mathrm{PO}$ holds true, we can easily explicitly write Eq. (3) in components as follows:

$$
v_{i}^{\mathbb{S}}(E)=\int_{\Lambda} E(\lambda) \sum_{j} A_{i j}^{\mathbb{S}} R_{j}(\lambda) d \lambda,
$$

which, thanks to Eq. (2), can be rewritten :

$$
\int_{\Lambda} E(\lambda)\left(S(\lambda) R_{i}(\lambda)-\sum_{j} A_{i j}^{\mathbb{S}} R_{j}(\lambda)\right) d \lambda=0 .
$$

The other assumption of $\mathrm{PO}$ is that the RM should be independent of the illuminant, i.e. Eq. (9) must be true for any 
$E(\lambda)$. Thanks to the Fundamental Lemma of Calculus of Variations [3], it follows that:

$$
S(\lambda) R_{i}(\lambda)-\sum_{j} A_{i j}^{\mathbb{S}} R_{j}(\lambda)=0, \quad \forall \lambda \in \Lambda .
$$

Thanks to Eq. (10), we can avoid performing a linear regression over a specified database of illuminants as in PO's method, because it can now be performed over $\Lambda$. We will call this approach the Illuminant Independent (II) approach.

\subsection{Quantitative comparison between PO's and the II approaches}

In our calculations we used the same databases as PO did for natural illuminants [4-6] and for Munsell chips [7], the latter made available by the University of Joensuu (http://spectral.joensuu.fi/). However, the database of natural surfaces that we used was taken from [8], Joensuu and Trieste Universities. For cone sensitivities, we used the $2^{\circ}$ Stockman \& Sharp cone fundamentals [9] as in [10] instead of the $10^{\circ}$ Stiles \& Burch [11] or the Smith \& Pokorny [12] color matching functions used in PO's and V-C's papers respectively. The reason for our choice is simply that Stockman \& Sharp cone fundamentals directly refer to the sensitivity of human photoreceptors. In any case, the results are similar for all of the sensor functions chosen among those three.

Similarly to PO [1], we checked the validity of the linear and illuminant-independent hypothesis by computing the empirical variance of the residual $\eta=y-\hat{y}$, where $y$ is the known quantity that the linear model tries to approximate and $\hat{y}$ is the approximated value obtained with the model. However, since we want the model to satisfy both Eq. (3) and Eq. (10), we considered the two kind of residuals defined in the following table:

\begin{tabular}{|c|c|c|}
\hline Residual & $y$ & $\hat{y}$ \\
\hline $\boldsymbol{\eta}_{1}$ & $S(\lambda) \cdot \mathbf{R}(\lambda)$ & $A^{\mathbb{S}} \mathbf{R}(\lambda)$ \\
\hline $\boldsymbol{\eta}_{2}$ & $\mathbf{v}^{\mathbb{S}}(E)$ & $A^{\mathbb{S}} \mathbf{u}(E)$, \\
\hline
\end{tabular}

where $\mathbf{R}(\lambda)=\left(R_{L}, R_{M}, R_{S}\right)(\lambda)$.

For further clarification, we used the exponents ${ }^{\mathrm{PO}}$ and ${ }^{\mathrm{II}}$ to differentiate the cases where the RMs have been computed with PO's or the II approach, respectively.

We will denote with $\mathrm{VA}_{i}^{k}$ the percentage of variance of data accounted for by the model, i.e.

$$
\mathrm{VA}_{i}^{k}=1-\left\langle\frac{\operatorname{var}\left(\boldsymbol{\eta}_{i}^{k}\right)}{\operatorname{var}\left(\mathbf{y}_{i}\right)}\right\rangle, \quad i=1,2, k=\mathrm{PO}, \mathrm{II},
$$

where \langle\rangle represents the average of the quantity inside w.r.t. the channels L,M,S. Figure 1 and Table 1 summarize the results obtained with the II approach. In both graphs of Figure 1 , the full and dashed lines represent the average and median value of $\mathrm{VA}_{i}^{k}$, respectively. The graph on the left refers to $\mathrm{VA}_{1}^{\mathrm{II}}$, while on the right we show that of $\mathrm{VA}_{2}^{\mathrm{II}}$.

The average values for the two graphs are $97 \%$ for $\mathrm{VA}_{1}^{\mathrm{II}}$ and $99.7 \%$ for $\mathrm{VA}_{1}^{\mathrm{II}}$. However, it is important to stress that the minimum attained by $\mathrm{VA}_{1}^{\mathrm{II}}$ is $50 \%$, while that of $\mathrm{VA}_{2}^{\mathrm{II}}$ is $92 \%$.
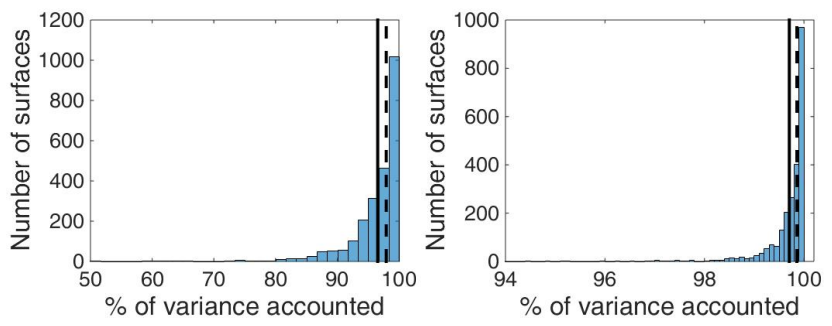

Fig. 1. Left: $\mathrm{VA}_{1}^{\mathrm{II}}$. Right: $\mathrm{VA}_{2}^{\mathrm{II}}$, for their definition, see (11).

The results for $\mathrm{VA}_{1}^{\mathrm{II}}$ show that, even though the linear and illuminant-independent hypothesis hold true for the large majority of the reflectance functions considered, they do not for all of them. However, the model still allows a very good approximation of the information about the reflected light $\mathbf{v}^{\mathbb{S}}(E)$, as shown by the results for $\mathrm{VA}_{2}^{\mathrm{II}}$. The explanation of this fact would require too much space and we will provide it in further communications.

Looking at Table 1 and comparing the $\mathrm{VA}_{2}^{\mathrm{II}}$ and $\mathrm{VA}_{2}^{\mathrm{PO}}$ obtained for natural illuminants, one could argue that the $A^{\mathbb{S}}$ computed with PO's approach allows better results than with the II approach. The reason for this is that, since the regression matrices $A^{\mathbb{S}}$ calculated with PO's strategy are computed with respect to a particular set of illuminants, natural in this case, they are optimally adapted to it, while those computed with the II approach are not. Thus, we thought it would be interesting to test the robustness of the two approaches with respect to illuminant changes i.e the consistency between the $A^{\mathbb{S}}$ matrices and the linear model in Eq. (3) when a drastic change of $E(\lambda)$ occurs. In order to test this, we considered two other illuminant datasets provided by Witzel et al in [10]. The first one was of fluorescent illuminants and the second was of almost-monochromatic visual lights. Both types of illuminants are very different from the natural ones.

In Table 1, we show the VA values with the II and PO approaches for natural, florescent and almost-monochromatic illuminants. The key point is that all values of $\mathrm{VA}_{2}^{\mathrm{PO}}$ were calculated with the RMs computed over the dataset of natural illuminants and applied to the fluorescent and almostmonochromatic conditions.

We notice that PO's matrices become less and less adapted to satisfy the linear model when we pass from the natural illuminant dataset to the florescent and the almostmonochromatic ones, with $\mathrm{VA}_{2}^{\mathrm{PO}}$ average values of $99.9 \%$ to $98.7 \%$ to $91.7 \%$ respectively. In particular, we observe the presence of a negative value for $\mathrm{VA}_{2}^{\mathrm{PO}}$. Instead, the $A^{\mathbb{S}}$ matrices computed with the II model remain, in terms of mean, 


\begin{tabular}{|c|c|c|c|c|c|c|c|c|c|}
\hline & \multicolumn{3}{|c|}{$\mathrm{VA}_{1}^{\mathrm{II}}$} & \multicolumn{3}{c|}{$\mathrm{VA}_{2}^{\mathrm{II}}$} & \multicolumn{3}{c|}{$\mathrm{VA}_{2}^{\mathrm{PO}}$} \\
\hline & mean & median & min & mean & median & min & mean & median & min \\
\hline natural & 96.57 & 97.95 & $\mathbf{5 0 . 2 5}$ & 99.70 & 99.96 & $\mathbf{9 1 . 9 3}$ & 99.94 & 99.97 & $\mathbf{9 8 . 8 3}$ \\
\hline fluorescent & $"$ & $"$ & $"$ & 99.45 & 99.75 & $\mathbf{8 7 . 2 3}$ & 98.69 & 99.45 & $\mathbf{6 5 . 5 3}$ \\
\hline monochromatic & $"$ & $"$ & $"$ & 96.33 & 97.83 & $\mathbf{3 7 . 7 2}$ & 91.68 & 96.00 & $\mathbf{- 2 . 3 0}$ \\
\hline
\end{tabular}

Table 1. VA values in the case of natural, florescent and almost-monochromatic illuminants. See text for further explanations.

median and minimal value, a better approximation than PO's for the florescent and almost-monochromatic sets.

Once computed, the RMs found with the II approach are more reliable than those found with PO's in terms of generalisability to other illuminants. They can thus serve as a starting point to find a unique change of basis transform independent with respect to the illuminant.

\section{A THEORETICAL AND COMPUTATIONAL DISCUSSION OF PO'S MODEL IN THE CONTEXT OF COMPUTER VISION}

In their article, $\mathrm{PO}$, and after them $\mathrm{V}-\mathrm{C}$, pointed out the possible link of the model with common approaches used in computational vision to achieve color constancy, such as the von Kries algorithm [13]. They argued that this link is valid if the matrices $A^{\mathbb{S}}$ can be diagonalized using a unique transformation that is independent of the surface $\mathbb{S}$. For the sake of clarity, we will start this section by describing thoroughly the bridge between PO's model and computer vision. Then, we will show that the existence of a $\mathbb{S}$-independent diagonalizing transformation is a theoretical consequence of PO's model applied to multiple surfaces. Finally, we will present a novel approach to compute this transformation without resorting to any standard illuminant or any particular illuminant database.

\subsection{Bridge between PO's model and computer vision}

Suppose that we have a visual scene illuminated by only one spatially homogeneous light source $E(\lambda)$, and consider an RGB camera with spectral sensitivity functions picked in the low, medium and high visible wavelengths, usually indicated with $\mathrm{R}, \mathrm{G}$ and $\mathrm{B}$, respectively. We denote the spectral sensitivity functions as $\rho_{c}: \Lambda \rightarrow \mathbb{R}^{+}, c \in\{R, G, B\}$. The analysis performed in [14] showed that $\operatorname{supp}\left(\rho_{c}\right)$, the supports of the functions $\rho_{c}$, are very similar and weakly overlapping compared to the spectral sensitivity functions of retinal cones.

In the Lambertian digital image formation model, see e.g. [15], the intensity $v_{c}(x, E)$ of a pixel $x$ in the chromatic channel $c$ is represented as follows:

$$
v_{c}(x, E)=\int_{\Lambda} \rho_{c}(x, \lambda) S(x, \lambda) E(\lambda) d \lambda .
$$

If we consider the supports $\operatorname{supp}\left(\rho_{c}\right)$ as not overlapping and the reflectance functions $S(x, \lambda)$ as constant with respect to $\lambda$ in each subset $\operatorname{supp}\left(\rho_{c}\right)$, i.e. $S(x, \lambda) \equiv S_{c}(x)$ for all $\lambda \in$ $\operatorname{supp}\left(\rho_{c}\right)$ and $c \in\{R, G, B\}$, then $v_{c}(x, E)$ can be re-written as:

$$
\left(\begin{array}{l}
v_{R}(x, E) \\
v_{G}(x, E) \\
v_{B}(x, E)
\end{array}\right)=\left(\begin{array}{ccc}
S_{R}(x) & 0 & 0 \\
0 & S_{G}(x) & 0 \\
0 & 0 & S_{B}(x)
\end{array}\right)\left(\begin{array}{l}
u_{R}(E) \\
u_{G}(E) \\
u_{B}(E)
\end{array}\right) .
$$

Notice that the simultaneous dependence on $x$ and $E$ of the left-hand side of the equation is separated into two multiplicative components, one being the diagonal matrix of elements $S_{c}$ which depends on $x$, and the other being the vector components $u_{c}$ which depend on $E$.

The von Kries diagonal transformation [13], used as a basis in several approaches to color constancy, see e.g. [16,17], is described by the following formula:

$$
\left(\begin{array}{l}
S_{R}(x) \\
S_{G}(x) \\
S_{B}(x)
\end{array}\right)=\left(\begin{array}{ccc}
1 / u_{R}(E) & 0 & 0 \\
0 & 1 / u_{G}(E) & 0 \\
0 & 0 & 1 / u_{B}(E)
\end{array}\right)\left(\begin{array}{l}
v_{R}(x) \\
v_{G}(x) \\
v_{B}(x)
\end{array}\right) .
$$

We stress that the von Kries matrix transformation is diagonal thanks to the diagonal nature of Eq. (13). Moreover, its independence with respect to the pixel position $x$ is a consequence of the separability property of Eq. (13).

We would like to translate what we have just stated into the terms of PO's model. First of all, the equivalent of Eq. (13) in PO's setting is Eq. (3). The matrices appearing in the two formulae depend only on surface properties. However $A^{\mathbb{S}}$ is not diagonal, thus it is not possible to write a von Kries-like transformation to obtain information on the reflectance.

Notice, however, that in the virtual basis expressed by Eq. (5) the RM is diagonal. If we write Eq. (5) in terms of components we find:

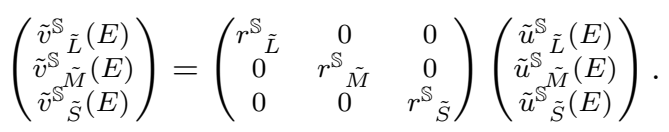

By direct computation, the previous formula can be re-written as:

$$
\left(\begin{array}{c}
r^{\mathbb{S}} \tilde{L} \\
r^{\mathbb{S}} \tilde{M} \\
r^{\mathbb{S}}
\end{array}\right)=\left(\begin{array}{ccc}
1 / \tilde{u}^{\mathbb{S}} \tilde{L}^{\mathbb{S}}(E) & 0 & 0 \\
0 & 1 / \tilde{u}_{\tilde{M}}^{\mathbb{S}}(E) & 0 \\
0 & 0 & 1 / \tilde{u}_{\tilde{S}}^{\mathbb{S}}(E)
\end{array}\right)\left(\begin{array}{c}
v^{\mathbb{S}} \tilde{L}(E) \\
v^{\mathbb{S}} \tilde{M}^{\tilde{M}}(E) \\
v^{\mathbb{S}} \tilde{S}(E)
\end{array}\right) .
$$

If we look at Eqs. (14) and (16) we see that in the latter the diagonal matrix depends on the surface $\mathbb{S}$, as a consequence of the dependence of $\tilde{\mathbf{u}}^{\mathbb{S}}=T^{\mathbb{S}} \mathbf{u}$ on $\mathbb{S}$. This argument shows that, 
if we want to be able to apply a von Kries-like transformation in PO's framework, the transformation $T^{\mathbb{S}}$ to the virtual basis must be independent of $\mathbb{S}$.

In the following subsections we will discuss this important issue in theoretical and computational terms.

\subsection{A theoretical argument for the existence of a unique virtual basis in the context of PO's framework}

The existence of a global change of basis transformation can be theoretically derived from Eqs. (1), (2) and (3), the very roots of PO's model. First of all, let us recall an important result of linear algebra: a set of matrices is simultaneously diagonalizable if and only if they commute. In particular, in PO's setting, this means that two matrices $A^{\mathbb{S}_{1}}$ and $A^{\mathbb{S}_{2}}$, relative to two arbitrary surfaces $\mathbb{S}_{1}$ and $\mathbb{S}_{2}$, can be diagonalized in the same basis if and only if they commute, i.e. $A^{\mathbb{S}_{1}} A^{\mathbb{S}_{2}}=A^{\mathbb{S}_{2}} A^{\mathbb{S}_{1}}$. We shall now give an argument to show that the commutativity of these matrices must indeed hold true. First of all, notice that, from a physical point of view, light reflected by a surface of spectral reflectance $S(\lambda)$ is indistinguishable from light emitted by a source whose power distribution is $E(\lambda) S(\lambda)$. Considering Eqs. (1) and (2), this can be translated into the following expression:

$$
\begin{aligned}
v_{i}^{\mathbb{S}}(E) & =\int_{\Lambda} R_{i}(\lambda)[S(\lambda) E(\lambda)] d \lambda, \quad i=L, M, S \\
& =u_{i}(S \cdot E) .
\end{aligned}
$$

Thus, if we consider the situation of a ray of light reflected by a surface $\mathbb{S}_{1}$ onto another surface $\mathbb{S}_{2}$, then due to the definition of reflectance [18] the accessible information about the light reflected in this process is:

$$
\begin{aligned}
u_{i}\left(S_{1} S_{2} \cdot E\right) & =\int_{\Lambda} R_{i}(\lambda)\left[S_{1}(\lambda) S_{2}(\lambda) E(\lambda)\right] d \lambda \\
& =\int_{\Lambda} R_{i}(\lambda)\left[S_{2}(\lambda) S_{1}(\lambda) E(\lambda)\right] d \lambda \\
& =u_{i}\left(S_{2} S_{1} \cdot E\right)
\end{aligned}
$$

which shows that the role of $\mathbb{S}_{1}$ and $\mathbb{S}_{2}$ can be inverted in the process described above.

If we write it in its vector form we find:

$$
\begin{aligned}
\mathbf{u}\left(S_{2} S_{1} \cdot E\right) & =\mathbf{v}^{\mathbb{S}_{2}}\left(S_{1} \cdot E\right)=A^{\mathbb{S}_{2}} \int_{\Lambda} \mathbf{R}(\lambda) S_{1}(\lambda) E(\lambda) d \lambda \\
& =A^{\mathbb{S}_{2}} A^{\mathbb{S}_{1}} \mathbf{u}(E) .
\end{aligned}
$$

Similarly, if we compute $\mathbf{u}\left(S_{1} S_{2} \cdot E\right)$, we obtain $A^{\mathbb{S}_{1}} A^{\mathbb{S}_{2}} u(E)$. Thanks to Eq. (18), we have $A^{\mathbb{S}_{1}} A^{\mathbb{S}_{2}} u(E)=A^{\mathbb{S}_{2}} A^{\mathbb{S}_{1}} u(E)$ for all pairs of surfaces $\mathbb{S}_{1}$ and $\mathbb{S}_{2}$, hence the commutativity of the RM matrices and the existence of a global change of basis transformation.

\subsection{A novel strategy to find a unique diagonalization ma- trix in PO's setting}

Now that we have provided an argument for the existence of a unique transformation $T$ able to jointly diagonalise all RM at once, we have to computationally find it.

This problem has already been considered by $\mathrm{V}-\mathrm{C}$ et al. in [2], where the authors proposed a strategy to find $T$ which, however, depends on the choice of a particular illuminant and, once again, on the database that was selected. Here we are going to describe a new strategy that does not have these limitations.

A commonly used measure for the the joint diagonality of a set of $N$ matrices $\left\{M^{k}\right\}_{k=1}^{N}$ proposed in [19] is the average of the squared Frobenius norm of the off-diagonal elements of each matrix, i.e.

$$
\begin{aligned}
\mathrm{JD} & =\frac{1}{N} \sum_{k=1}^{N} \sum_{i \neq j}\left|M_{i j}^{k}\right|^{2} \\
& =\frac{1}{N} \sum_{k=1}^{N}\left\|\left[M^{k}-\operatorname{diag}\left(M^{k}\right)\right]_{i j}\right\|_{\mathrm{FRO}}^{2}
\end{aligned}
$$

The larger $J D$, the less the set of matrices is jointly diagonal.

The goal of the algorithm that we want to propose is thus to find the optimal change of basis transformation $T_{\mathrm{opt}}$ that minimizes $J D$. Using the notations introduced for PO's framework in the case of $N$ surfaces $\mathbb{S}_{k}$, we can write the optimality condition as follows:

$$
\begin{aligned}
T_{\mathrm{opt}} & =\underset{T \in \mathbb{R}^{3 \times 3}}{\arg \min } \mathrm{JD}(T) \\
& =\underset{T \in \mathbb{R}^{3 \times 3}}{\arg \min } \frac{1}{N} \sum_{k=1}^{N} \sum_{i \neq j}\left|\left(T^{-1} A^{\mathbb{S}_{k}} T\right)_{i j}\right|^{2} .
\end{aligned}
$$

We stress that the matrices that appear in the previous equation are not symmetric nor self-adjoint, thus we cannot apply the minimization strategies proposed in [19-23] in our context. However, the gradient descent strategy proposed by (Hori, 1999) [24] can be adapted to our framework because it requires only the non-singularity of matrices.

The gradient of $J D$ is:

$\nabla \operatorname{JD}(T)=2 T \sum_{k=1}^{N}\left[\left(T^{-1} A^{\mathbb{S}_{k}} T\right)^{t},\left(T^{-1} A^{\mathbb{S}_{k}} T-\operatorname{diag}\left(T^{-1} A^{\mathbb{S}_{k}} T\right)\right)\right]$,

where $\left[M_{1}, M_{2}\right]=M_{1} M_{2}-M_{2} M_{1}$ is the commutator between matrices, so the discrete gradient descent equation to find $T_{\text {opt }}$ is:

$$
T_{n+1}=T_{n}-\alpha_{n} \frac{\nabla \mathrm{JD}\left(T_{n}\right)}{\left\|\nabla \mathrm{JD}\left(T_{n}\right)\right\|}, \quad n \geq 0
$$

where $0<\alpha_{n}<1$ is the step dependent weight of the numerical scheme. 


\begin{tabular}{|cc|cc|cc|}
\hline \multicolumn{2}{|c|}{ Stockman \& Sharp } & \multicolumn{3}{c|}{ Smith \& Pokorny } & \multicolumn{3}{c|}{ Stiles \& Burch } \\
$\left(\begin{array}{cccc}1.6934 & -1.5335 & 0.0750 \\
-0.8547 & 2.1269 & -0.2243 \\
0.0215 & -0.0432 & 1.0169\end{array}\right)$ & $\left(\begin{array}{cccc}17191 & -1.5603 & 0.0666 \\
-0.8836 & 2.1573 & -0.1800 \\
0.0349 & -0.0680 & 1.0128\end{array}\right)$ & $\left(\begin{array}{ccc}1.0388 & -0.3447 & 0.1559 \\
-0.1066 & 1.0896 & -0.1059 \\
0.0043 & -0.0137 & 1.0127\end{array}\right)$ \\
\hline
\end{tabular}

Table 2. Optimal global change of basis matrix found with the gradient descent for the three kinds of cone sensitivities used in [1], [2], [10].

The matrix which minimizes the error JD, among the 2340 matrices of our dataset with columns given by the eigenvectors of $\left\{A^{\mathbb{S}_{k}}\right\}$, was selected as the initial transformation $T_{0}$.

The stopping criterion of the numerical scheme is the following: we consider that the algorithm converged when the $L^{1}$ distance between $T_{n+1}$ and $T_{n}$ is less than $\varepsilon=10^{-3}$. This value for $\varepsilon$ was chosen empirically on the basis of the numerical values of the matrix elements of $T_{n}$, as well as for the efficiency of the algorithm.

In Fig. 2 we show the decreasing behavior of $\operatorname{JD}\left(T_{n}\right)$ with respect to $n$. It can be seen that, after 6 iterations, the corrections are negligible. Thus, in practice, we can considere, the algorithm to be convergent after 6 iterations with a value of JD of 0.0006 . This value is such that ratio between the squared Frobenius norm of the off-diagonal elements and that of the whole matrices, averaged over the entire dataset, accounts for less than $0.4 \%$.

Table 2 shows the $T_{\text {opt }}$ found with the gradient descent for the three kinds of cone sensitivities.

\section{CONCLUSION}

We have shown in this work that an alternative method to PO's can be used to compute the reflectance matrices $A^{\mathbb{S}}$. This method has the advantage of being fully independent with respect to the illuminant. It is thus more robust to illuminant

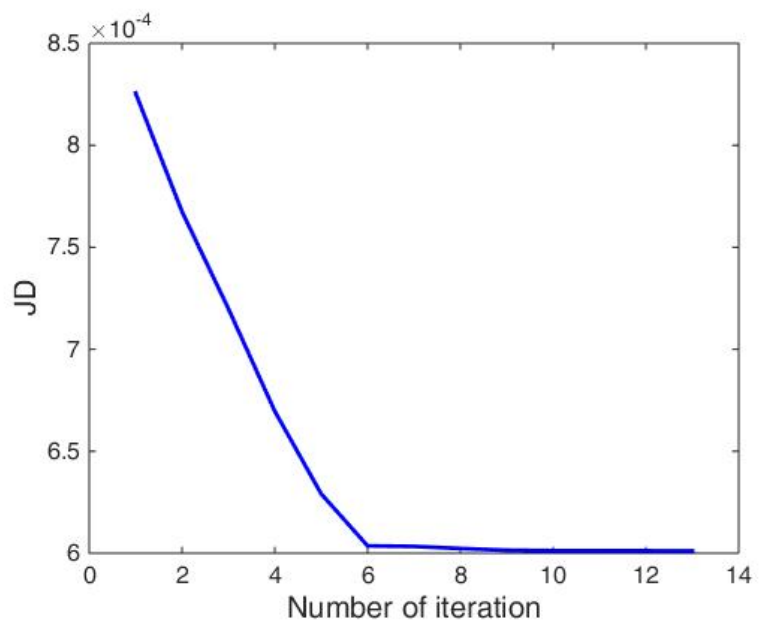

Fig. 2. Gradient descent applied on the set of 2340 RMs computed using the II approach. change, while still allowing satisfactory results in the approximation of the information available to the eye about the reflected light $\mathbf{v}^{\mathbb{S}}(E)$. It also allows the computation, through a simple gradient descent, of a global change of basis transformation $T$ which is independent of illuminants, and compatible with classic von Kries-like approaches to achieve color constancy despite the significant overlap of human cone sensitivity functions.

\section{ACKNOLEDGEMENT}

This work was financed by ERC Advanced grant "FEEL" Number 323674 to J. K. O’Regan

\section{REFERENCES}

[1] D. L. Philipona and J K. O'Regan, “Color naming, unique hues, and hue cancellation predicted from singularities in reflection properties," Visual neuroscience, vol. 23, no. 3-4, pp. 331-339, 2006.

[2] J. Vazquez-Corral, J K. O'Regan, M. Vanrell, and G. D Finlayson, "A new spectrally sharpened sensor basis to predict color naming, unique hues, and hue cancellation," Journal of vision, vol. 12, no. 6, pp. 7-7, 2012.

[3] I. M. Gelfand, R. A. Silverman, et al., Calculus of variations, Courier Corporation, 2000.

[4] J. Romero, A Garcia-Beltrán, and J. Hernández-Andrés, "Linear bases for representation of natural and artificial illuminants," JOSA A, vol. 14, no. 5, pp. 1007-1014, 1997.

[5] Deane B Judd, David L MacAdam, Günter Wyszecki, HW Budde, HR Condit, ST Henderson, and JL Simonds, "Spectral distribution of typical daylight as a function of correlated color temperature," Josa, vol. 54, no. 8, pp. 1031-1040, 1964.

[6] C-C. Chiao, T. W. Cronin, and D. Osorio, "Color signals in natural scenes: characteristics of reflectance spectra and effects of natural illuminants," JOSA A, vol. 17, no. 2, pp. 218-224, 2000.

[7] J. PS Parkkinen, J Hallikainen, and T Jaaskelainen, "Characteristic spectra of munsell colors," JOSA A, vol. 6, no. 2, pp. 318-322, 1989. 
[8] S. Westland, J. Shaw, and H. Owens, "Colour statistics of natural and man-made surfaces," Sensor Review, vol. 20, no. 1, pp. 50-55, 2000.

[9] A. Stockman and L. T Sharpe, "The spectral sensitivities of the middle-and long-wavelength-sensitive cones derived from measurements in observers of known genotype," Vision research, vol. 40, no. 13, pp. 1711-1737, 2000.

[10] C. Witzel, F. Cinotti, and J. K. O'Regan, "What determines the relationship between color naming, unique hues, and sensory singularities: Illuminations, surfaces, or photoreceptors?"' Journal of vision, vol. 15, no. 8, pp. 19-19, 2015.

[11] W. S. Stiles and J. M Burch, "Npl colour-matching investigation: final report (1958)," Journal of Modern Optics, vol. 6, no. 1, pp. 1-26, 1959.

[12] V. C Smith and J Pokorny, "Spectral sensitivity of the foveal cone photopigments between 400 and $500 \mathrm{~nm}$," Vision research, vol. 15, no. 2, pp. 161-171, 1975.

[13] J. von Kries, "Chromatic adaptation," Festschrift der Albrecht-Ludwigs-Universität, pp. 145-158, 1902.

[14] J. Jiang, D. Liu, J. Gu, and S. Susstrunk, "What is the space of spectral sensitivity functions for digital color cameras?," 2013, pp. 168-179.

[15] T. Gevers, A. Gijsenij, J. Van de Weijer, and J-M Geusebroek, Color in computer vision: fundamentals and applications, vol. 23, John Wiley \& Sons, 2012.

[16] G. West and M. H Brill, "Necessary and sufficient conditions for von kries chromatic adaptation to give color constancy," Journal of Mathematical Biology, vol. 15, no. 2, pp. 249-258, 1982.

[17] D. A Forsyth, "A novel algorithm for color constancy," International Journal of Computer Vision, vol. 5, no. 1, pp. 5-35, 1990.

[18] Thermal insulation Heat transfer by radiation Physical quantities and definitions, ISO 9288:1989 ISO catalogue, 1989.

[19] A. Bunse-Gerstner, R. Byers, and V. Mehrmann, "Numerical methods for simultaneous diagonalization," SIAM Journal on Matrix Analysis and Applications, vol. 14, no. 4, pp. 927-949, 1993.

[20] J-F. Cardoso and A. Souloumiac, "Jacobi angles for simultaneous diagonalization," SIAM J. Mat. Anal. Appl., vol. 17, no. 1, pp. 161-164, Jan. 1995.
[21] B. Afsari and P. S Krishnaprasad, "Some gradient based joint diagonalization methods for ica," in Independent Component Analysis and Blind Signal Separation, pp. 437-444. Springer, 2004.

[22] A. Ziehe, P. Laskov, G. Nolte, and K-R. Müller, “A fast algorithm for joint diagonalization with non-orthogonal transformations and its application to blind source separation," The Journal of Machine Learning Research, vol. 5, pp. 777-800, 2004.

[23] P. Tichavskỳ and A. Yeredor, "Fast approximate joint diagonalization incorporating weight matrices," Signal Processing, IEEE Transactions on, vol. 57, no. 3, pp. 878-891, 2009.

[24] G Hori, "Joint diagonalization and matrix differential equations," Proc. of NOLTA99, pp. 675-678, 1999. 